\title{
Public's Knowledge, Attitude and Practice towards COVID-19 in the Normalization Stage of Plague Prevention and Control: A Cross-sectional Survey in China
}

\author{
Junlan Yan", Minna Zhang ${ }^{1}$, Xueyan Liu ${ }^{2, ~ *, ~ P e i r u ~ Z h o u, ~, ~, ~ J i e w e i ~ H u a n g ~}{ }^{2}$, Kaiming Ye \\ ${ }^{1}$ Nursing Institute, Jinan University, Guangzhou, China \\ ${ }^{2}$ Department of Endocrine Metabolism, The First Affiliated Hospital of Jinan University, Guangzhou, China

\section{Email address:} \\ 15528436168@163.com (Junlan Yan), zhangminna@stu2018.jnu.edu.cn (Minna Zhang), yhlu@163.com (Xueyan Liu), \\ luzhmm@126.com (Peiru Zhou) \\ ${ }^{*}$ Corresponding author
}

\section{To cite this article:}

Junlan Yan, Minna Zhang, Xueyan Liu, Peiru Zhou, Jiewei Huang, Kaiming Ye. Public's Knowledge, Attitude and Practice towards COVID-19 in the Normalization Stage of Plague Prevention and Control: A Cross-sectional Survey in China. American Journal of Nursing Science. Vol. 10, No. 1, 2021, pp. 1-7. doi: 10.11648/j.ajns.20211001.11

Received: December 6, 2020; Accepted: December 23, 2020; Published: January 4, 2021

\begin{abstract}
Background: COVID-19 has been declared a global public health emergency, posing a serious threat to the life and health of the general public. So the aim of this study is to investigate the ability of plague prevention of residents about COVID-19 in the normalization stage of epidemic prevention and control in China, thereby providing reference information for relevant institutions to formulate targeted health education measures. Methods: We designed the KAP for COVID-19 Prevention and Control questionnaire partly based on the COVID-19 prevention and control guidelines published by the National Health Commission of China. The questionnaire was distributed through the Questionnaire Star online survey platform between June 28 and June 31, 2020. Convenience and snowball sampling methods were used to collect data. The questionnaire link was first sent to subjects through online social platforms such as WeChat and QQ, where they were invited to send the link to their families, colleagues, and friends. Results: The survey involved 314 members of the public. Questionnaire scores were $9.90 \pm 1.967$ (range: 0 14), $22.97 \pm 2.90$ (range: 5 25), and 37.64 \pm .30 (range: 9 45) for participant COVID-19 prevention and control knowledge, attitude and practice, respectively. More than $80 \%$ of the participants had good knowledge of the core symptoms, incubation and isolation periods, and three major preventive measures of COVID-19, but only $31.8 \%$ of them answered correctly on the main transmission routes of COVID-19. There were errors when they were asked about the treatment measures, with $27.7 \%$ of participants believing that using Vedicilin (an oral antiviral liquid) and gargling brackish water can prevent COVID-19. More than $95 \%$ of participants believed that the normalization stage of epidemic prevention still requires personal protective measures. Of the participants, $60 \sim 64 \%$ were able to perform basic preventive behaviors such as wearing a mask when going out, washing hands at home, and going out less frequently, $52.6 \%$ could always comply with cough etiquette, and $40.8 \%$ could always follow the seven-step hand washing method. Conclusions: During the normalization stage of the COVID-19 plague prevention, the public had incomplete knowledge on latest prevention strategies Although belief was evident, behavioral compliance still needs to be improved. Relevant government departments or medical institutions need to relay relevant knowledge to those in blind spots, and to guide the public to maintain participation in effective and science-based prevention of the novel coronavirus.
\end{abstract}

Keywords: Novel Coronavirus, COVID-19, Knowledge, Attitude, Practice

\section{Introduction}

An outbreak of COVID-19, which is a disease caused by a novel coronavirus (SARS-CoV-2), had quickly spread to many countries by the end of December 2019. The World Health
Organization announced on March 11, 2020 that COVID-19 was considered a global pandemic [1,2]. COVID-19 epidemic situation occurred in December 2019 is a big public health emergency, which has brought huge challenges to health and epidemic prevention due to its rapid spread, wide infection area 
and difficult prevention and control. Governments around the world quickly implemented a variety of public health and behavioral interventions when the epidemic broke out, including lockdown and containment strategies, testing, contact tracing, strengthening the medical system, and distributing personal protective equipment [3]. In June 2020, the epidemic in China had almost been eliminated, and was in the normalized epidemic prevention and control stage. The focus of epidemic prevention was to guard against the risk of imported cases from abroad and sporadic local cases [4].

The Middle East respiratory syndrome coronavirus was first discovered in Saudi Arabia in 2012. In the following years, it recurred as intermittent sporadic cases, community clusters, and hospital outbreaks [5]. Therefore, the focus of epidemic prevention has become preventing sporadic clusters of cases. Today, an effective vaccine is still not widely available. The incidence of respiratory infectious diseases is high during autumn and winter. This worsens the situation, requiring every citizen to take part in normalized prevention and control measures. The knowledge, attitude and practice (KAP) of the public in coping with respiratory infectious diseases is of vital importance [6]. KAP theory holds that human behavior change includes three continuous processes: the acquisition of correct knowledge, the generation of positive beliefs and the adoption of behavior (or practice), involving a series of beliefs about the etiology, symptom identification, effective treatments and consequences of the disease [7, 8]. The low level of knowledge and attitude towards infectious diseases can aggravate the panic degree of the population and will not be conducive to curbing the spread of the epidemic [9]. At the same time, the KAP investigation helps to provide useful information on epidemic prevention and control measures, and Kobayash and his colleagues report that the health awareness and beliefs conveyed by KAP related to Ebola can be used as a basis for the development of public health education measures about Ebola [10]. In the uncertainty of this epidemic, the public's KAP to COVID-19 is the key to delay the spread of the epidemic. At the beginning of the outbreak, scholars from multiple countries investigated the KAP levels among residents in different areas of novel coronavirus protection [11-13]. It is vital to consider the public's prevention and control capabilities at diverse stages, since the public's KAP change with the epidemic of the disease, preventive fatigue may develop over time, which can be achieved through subsequent investigations [3].

The present study aimed to determine the self-prevention and control capabilities of the general public during the normalization stage of epidemic prevention and control, which will help relevant agencies make precise adjustments to these measures.

\section{Material and Methods}

\subsection{Study Subjects}

A cross-sectional survey was conducted, applying convenience and snowball sampling methods to invite
Chinese residents who use social media and smart phones to voluntarily complete a questionnaire. Data were collected using the Questionnaire Star online survey tool. The survey was conducted between June 28 and June 31, 2020. The questionnaire was sent to the participants through social media, also encouraging them to send it to their friends and relatives. To assure the validity of the survey, each IP address could only respond once. Participants had to answer every question. Otherwise they could not submit their responses, and were advised to take at least 180 seconds per question.

\subsection{Study Instruments and Scoring Method}

An investigation instrument was designed based on guidelines for the COVID-19 epidemic and other information on prevention.

The instrument consists of four main dimensions: (1) demographics, which include sociodemographic information such as gender, race, place of residence, education level, occupation, and income; (2) knowledge about COVID-19, which was tested using 14 multiple-choice questions, including knowledge of COVID-19 symptoms, incubation period, transmission methods, and preventive measures; (3) attitude toward COVID-19, which comprised 5 items relating to the issue of continuing to implement prevention and control behaviors despite being in the normalization stage of epidemic prevention and control; and (4) the behavior dimension, which includes how the public implement epidemic prevention measures, such as reducing going out, wearing masks, and washing hands at home.

The outline for the general scoring method used for the KAP questionnaire is as follows: (1) each item in the knowledge dimension received a score of " 1 " (correct answer) or " 0 " (incorrect answer), with a total score between 0 and 14; (2) for each item of the attitude dimension, a 5-point Likert scale ranging from "very much disagree" (1 point) to "very much agree" ( 5 points) was used, with a total score between 5 and 25; and (3) a 5-point Likert scale was also used for each item in the behavior dimension, ranging from "never" (1 point) to "always" ( 5 points), with a total score between 9 and 45 points. Each dimension level depends on the corresponding dimension score.

\subsection{Data Processing and Analysis}

Frequency and composition ratios were utilized to represent the participant's demographic and sociological data, and the overall KAP score was represented using mean \pm standard deviation. KAP dimension items were represented by their composition ratios. The relationship between KAP was analyzed using Pearson's correlation coefficient. Statistical significance was defined as $P<0.05$.

\section{Results}

\subsection{Demographic Characteristics of Participants}

The questionnaire was completed by 314 participants from 18 Chinese provinces. Among them, $42.7 \%$ of the participants 
$(n=134)$ were male and $57.3 \%(n=180)$ were female, $96.8 \%$

had an education level of a college degree or higher (Table 1).

$(n=304)$ identified as having Han ethnicity, and $52.9 \%(n=166)$

Table 1. Sociodemographic characteristics of the participants.

\begin{tabular}{llll}
\hline Variable & N (\%) & Variable & N (\%) \\
\hline Gender & & Marriage & $106(33.8)$ \\
Male & $134(42.7)$ & Single & $208(66.2)$ \\
Female & $180(57.3)$ & Married & \\
Nation & & Occupation & $72(22.9)$ \\
Han nationality & $304(96.8)$ & Farmer & $48(15.3)$ \\
Minority & $10(3.2)$ & Student & $22(7.0)$ \\
Age (years) & & Worker & $39(12.4)$ \\
$<18$ & $8(2.5)$ & Medical workers & $133(42.4)$ \\
$18 \sim 49$ & $245(78.0)$ & Other & $167(53.2)$ \\
$50 \sim 64$ & $51(16.2)$ & Monthly income & $80(25.5)$ \\
$\geq 65$ & $10(3.2)$ & $\leq 1999$ & $54(17.2)$ \\
Place of residence & & $2000-4999$ & $13(4.1)$ \\
City & $159(50.6)$ & $5000-9999$ & $\geq 10000$ \\
Township & $155(49.4)$ & & \\
Education & & & \\
Technical secondary school and below & $166(52.9)$ & & \\
College degree and above & $148(47.1)$ & & \\
\hline
\end{tabular}

*Single status includes unmarried, divorced, widowed.

\subsection{KAP Scores for COVID-19 in the Dimensions of Prevention and Control}

The score for each dimension of the questionnaire did't get a skewed distribution. The average total score for the Knowledge was $9.90 \pm 1.95$, Attitude was $22.97 \pm 2.90$, and Practice was $37.64 \pm 5.30$. The score was standardized as percentages $(($ actual score $) /($ highest possible score $) \times 100)$.
Score of $\geq 85 \%, 60 \sim 85 \%$, and $\leq 60 \%$ were considered to indicate good, moderate, and low levels of KAP, respectively. Approximately one-quarter (23.3\%) of participants had a good level of awareness, 79\% had a positive attitude toward the prevention and control of the novel coronavirus, and $48.7 \%$ tended to practice preventive behaviors (Table 2).

Table 2. COVID-19 prevention and control of KAP in various dimensions.

\begin{tabular}{llll}
\hline \multirow{2}{*}{ Dimension } & Score $($ Means \pm S D $)$ & COVID-19 protection level $(\mathbf{n}) \%$ & Medium level \\
\cline { 2 - 4 } & & Low level & $167(53.2)$ \\
\hline knowledge & $9.90 \pm 1.95$ & $74(23.6)$ & $58(18.5)$ \\
attitude & $22.97 \pm 2.90$ & $8(2.5)$ & $150(47.8)$ \\
practice & $37.64 \pm 5.30$ & $11(3.5)$ & $248(79.0)$ \\
\hline
\end{tabular}

\subsection{Knowledge of Participants on COVID-19}

More than $80 \%$ of participants had knowledge of the three core symptoms of COVID-19 (fever, cough, and dyspnea), the time required for close contacts to isolate (14 days), the incubation period (7 14 days), and the three preventive measures (wash your hands as soon as you get home, wear a mask when you go out, and avoid going to places with large crowds where possible). More than $50 \%$ of the participants were able to accurately identify who are susceptible. Correct medicines, only $31.8 \%$ of the participants were fully aware of the main transmission route for COVID-19 (Table 3).

Table 3. Responses of participants to questions on knowledge about COVID-19.

\begin{tabular}{llr}
\hline Question & Correct Answer Number & Correct Answer Rate (\%) \\
\hline 1. What are the primary symptoms of COVID-19? & 312 & 99.4 \\
2. Which of the following people are susceptible to the new coronavirus? & 160 & 306 \\
3. How many days do close contacts of COVID-19 need medical isolation? & 263 \\
4. How many days is the incubation period for COVID-19? & 100 \\
5. What are the major routes of transmission of COVID-19? & 204 \\
6. Which of the following drugs can treat COVID-19? & 227 \\
7. Can you prevent COVID-19 by gargling with brackish water, taking antiviral oral liquid, & 37.5 \\
and using vinegar fume? & 259 \\
8. Novel Coronavirus spreads through droplets should your home be closed? & 175 \\
9. Which method can indoor disinfection take below? & 185 \\
10. Which of the following three behaviors are true when coughing or sneezing? & 265 \\
11. What are the three main preventive measures for COVID-19? & 235 \\
12. Regarding the use of masks, which of the following statements is incorrect? & 247 \\
13. Can used masks be cleaned before use? & 72.8 \\
14. How to dispose of used masks? & 82.5 \\
\hline
\end{tabular}




\subsection{Attitude Toward COVID-19}

Participants were positive and optimistic about the prevention and control of COVID-19. Most (92.7\%) of the participants believed that self-prevention learning should be carried out every day. More than $95 \%$ of the participants believed that in the regular phase of epidemic prevention and control, individual defense measures such as wearing masks, washing hands frequently, and reducing time spent outside of the home should remain the focus. Most (90.1\%) of the participants expressed that they are very willing to be a volunteer to support the anti-epidemic front.

\subsection{Practice Related to COVID-19}

Of the participants in this study, 60-64.1\% reported that they always wore a mask outside of the home, and washed their hands before every meal and as soon as they got home. Only about $30 \%$ of participants always learned. COVID-19's knowledge of epidemic prevention initiatively, and rarely went out, except to go to work and buy necessities (Table 4).

Table 4. Responses of participants to questions on practices toward COVID-19.

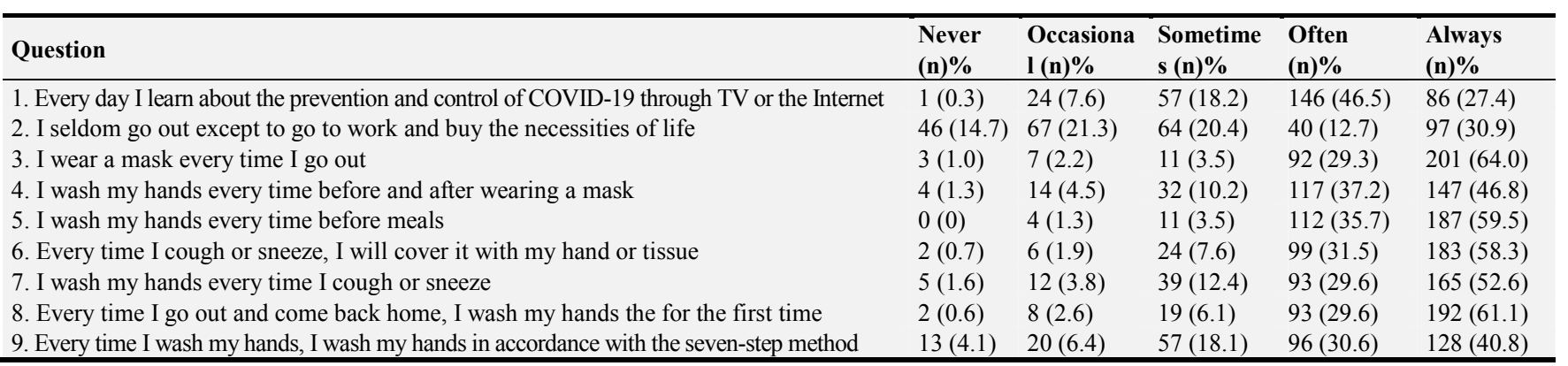

\subsection{Relationships of the KAP of COVID-19}

There were pairwise correlations among knowledge, attitude, and behavior, with coefficients of $0.221,0.174$, and 0.411, respectively. Each coefficient was statistically significant $(P<0.01)$.

\section{Discussion}

COVID-19 is a new virus that has spread rapidly to greatly affected the socioeconomic environment worldwide. Each epidemic has unique characteristics in terms of developmental process and control measures. The evolution process and prophylactic measures of all epidemic situations have their own traits, and providing epidemic prevention education is vital to curbing the spread [14]. Educational information should be adjusted to account for changing behavior patterns, which requires an understanding of the KAP levels of the public toward COVID-19 at different stages of epidemic prevention.

\subsection{The Public's Knowledge of Prevention and Control of COVID-19}

The participants had a moderate level of knowledge for the prevention and control of COVID-19, which is lower than in similar studies [15-17]. This is probably because those studies were conducted in the early stages of the outbreak where people paid more attention to the disease, or they had different definitions of awareness rates than in our study.

Further analysis of the prevention and control knowledge items revealed that the respondents have a high awareness rate of the three core symptoms of COVID-19, its incubation period, the three preventive measures, and cough etiquette.
Similar findings were reported in research surveys by Redd et al. [3] and Geldsetzer et al. [11]. However, only 51\% of participants could recognize people who are more susceptible to the COVID-19. $37.3 \%$ of participants were aware that novel coronavirus is more likely to infect the elderly. Similarly, Huifang et al. [18] and Hezima et al. [19] revealed that some people also agreed with this, which needs to be addressed since they may underestimate the risks and reduce the implementation of preventive measures. Only $31.8 \%$ of participants fully understood the transmission route of the novel coronavirus. A study in Malaysia found that $56.9 \%$ of the population did not know that the virus was transmitted through droplets [16]. This might be because $87.6 \%$ of participants were nonmedical personnel, who might find this knowledge to be too technical and difficult to understand [18, 20, 21]. Easy-to-understand educational methods (e.g., animated video and live teaching) should be adopted to increase the understanding of relevant knowledge.

In addition, $45.2 \%$ of participants had not learnt how to treat used masks. A similar Iranian survey indicated that $36.2 \%$ of respondents also did not know how to treat used masks [22]. As the epidemic enters the normalization stage of prevention and control, the public will continue to wear masks and a large number of masks will be discarded. Advising on disposal methods of discarded masks has become a necessary task to guide the public in avoiding secondary pollution.

A small proportion $(27.8 \%)$ of participants in this study believed that using Vedicilim, antiviral oral liquid, and brackish water gargle could prevent novel coronavirus infection. According to other similar studies, some people believe that washing the nasal cavity with salt water and eating garlic could prevent novel coronavirus infection [11, 23]. This shows that survey respondents had certain 
misunderstandings about protecting against COVID-19. This may be due to more than half of the interviewees lacking the education levels necessary to identify the correct epidemic prevention knowledge. Incorrect information might lead to indeterminacy and the public adopting the wrong preventive actions [24].

These findings demonstrate that the lack of knowledge on the prevention and control of the COVID-19 epidemic is related to a lack of judgement about false information. This highlights the importance of further strengthening the spread of epidemic prevention knowledge, especially for areas with low education. In addition, relevant health departments should strengthen the supervision of network platforms to clear and rectify rumors quickly, and assist the public in acquiring correct knowledge, guiding them to strengthen their sense of personal protection.

\subsection{The Public's Attitude and Practice of Prevention and Control of COVID-19}

The public had a positive attitude toward the prevention and control of COVID-19. More than $95 \%$ of participants believed that self-prevention measures should be taken. The vast majority of participants expressed their willingness to become an antiepidemic worker once the epidemic needs, which reflected the antiepidemic stance among the general public.

However, this study found that less than half of the participants $(48.7 \%)$ performed self-protection behaviors well, which needs to be further improved. This study had fewer participants (60-64.1\%) who would always implement three basic preventive measures (wearing a mask when going out, washing hands as soon as returning home, and going out less frequently) compared with other similar studies $[25,26]$.

Public compliance decreased over time due to mental exhaustion from epidemic information [27]. It is therefore necessary to better understand and target the management of the psychological states of the public at different stages of epidemic prevention and control, in order to avoid public fatigue from prevention and control.

Only $52.6 \%$ of participants reported that they always washed their hands after coughing or sneezing. Handwashing is not adopted by $44 \%$ of medical workers in Pakistan after coughing/sneezing [28]. This may occur because people believe that covering their faces with elbows or tissues when coughing or sneezing could prevent pathogens from contaminating their hands. Less than half of participants wash their hands before and after wearing a mask and use the seven-step handwashing method when doing so. In India, $44.6 \%$ of medical imaging students are unfamiliar with the seven-step handwashing method [23]. These phenomena confirm that the citizens have not mastered timing and seven-step method of handwashing. The local hygiene department should further the education system to improve knowledge on hand hygiene. At this stage, we must continue to enhance and implement public epidemic prevention, consolidate and sustain previous achievements in epidemic prevention.

\subsection{Correlation Analysis of KAP for COVID-19 Prevention and Control}

The present study found that those with a higher COVID-19 knowledge score had a greater likelihood of a positive attitude toward COVID-19 and preventive behavior, which is consistent with the findings of previous studies [12]. Sari et al. [29] showed that knowledge of and attitude toward COVID-19 were positively correlated. Peng et al. [21] indicated that the attitude and behavior toward COVID-19 were positively correlated. These results indicate that good knowledge of COVID-19 could induce more favorable public responses to the outbreak. Expanding knowledge on epidemic prevention and increasing public faith in self-protection is vital to improve protective behavior compliance.

\section{Conclusion}

The results of this study demonstrate that the public has moderate-level knowledge about and prevention practices for the epidemic situation. Further improving the level of public epidemic prevention and control is necessary despite the positive attitudes. While the participants had good basic knowledge of the prevalence, knowledge on transmission and drug treatment was lacking, and there were certain cognitive misunderstandings. It is recommended that during the regular prevention and control stage and the flu season, diversified forms of education should be adopted based on the public's knowledge of epidemic prevention (using platforms such as WeChat, QQ, small programs to push epidemic prevention, animation videos, posters, and manuals) to help the public better grasp the knowledge of epidemic prevention. Education on knowledge shortcomings and blind spots, especially the timing and seven-step method of handwashing, should be strengthened so that the public implement accurate epidemic prevention measures, and effectively prevent the novel coronavirus by combating the threat of various infectious diseases in fall and winter. The Global COVID-19 epidemic is not over yet, and public health education is still an effective measure to prevent and control the spread of the epidemic. This study shows that the level of knowledge, attitude and behavior of the public is different in different stages of epidemic prevention and control. Therefore, in the next epidemic prevention work, public health departments need to dynamically evaluate public knowledge, attitude and practice, expand the sample volume of research, and provide more COVID-19's scientific protection suggestions and measures. In order to formulate targeted and operable health education plans and policies to further enhance the protective ability of the public COVID-19.

\section{Limitations}

This study was subject to some limitations. The sample was of insufficient size and was not random. The research conclusions might be unreliable since a large proportion of the participants were aged 18 to 49 years and of Han 
ethnicity, and the degree of risk varied markedly within the study region. Therefore, future studies need to include network survey samples that are more representative.

\section{Author Contributions}

Junlan Yan and Minna Zhang are co-first authors.

\section{Conflict of Interest}

All the authors do not have any possible conflicts of interest.

\section{Acknowledgements}

The authors gratefully acknowledge the financial supports by the Natural Science Foundation of Guangdong Province of China under Grant numbers 2016B010108008.

\section{References}

[1] Cutler, D. (2020). How Will COVID-19 Affect the Health Care Economy? Jama, 323 (22), 2237-2238.

[2] Huang, C., Wang, Y., Li, X., Ren, L., Zhao, J., Hu, Y.,... \& Cheng, Z. (2020). Clinical features of patients infected with 2019 novel coronavirus in Wuhan, China. The lancet, 395 (10223), 497-506.

[3] Reddy, S. P., Sewpaul, R., Mabaso, M., Parker, S., Naidoo, I., Jooste, S., Mokhele, T., Sifunda, S., \& Zuma, K. (2020). South Africans' understanding of and response to the COVID-19 outbreak: An online survey. South African medical journal Suid-Afrikaanse tydskrif vir geneeskunde, 110 (9), 894-902

[4] National Health Commission of the people's Republic of China. The press conference of the Joint Prevention and Control Mechanism of the State Council on April 13, 2020 introduced the import of epidemic prevention and control abroad in accordance with the law. [EB/OL]. [2020-03-29]. Http://www.nhc.gov.cn/wjw/xwfbh/xwzb.shtml

[5] Hui, D. S., Azhar, E. I., Madani, T. A., Ntoumi, F., Kock, R., Dar, O.,... \& Zumla, A. (2020). The continuing 2019-nCoV epidemic threat of novel coronaviruses to global health-The latest 2019 novel coronavirus outbreak in Wuhan, China. International Journal of Infectious Diseases, 91, 264-266.

[6] Abdelhafiz, A. S., Mohammed, Z., Ibrahim, M. E., Ziady, H. H., Alorabi, M., Ayyad, M., \& Sultan, E. A. (2020). Knowledge, Perceptions, and Attitude of Egyptians Towards the Novel Coronavirus Disease (COVID-19). Journal of community health, 45 (5), 881-890.

[7] Fan, Y., Zhang, S., Li, Y., Li, Y., Zhang, T., Liu, W., \& Jiang, H. (2018). Development and psychometric testing of the Knowledge, Attitudes and Practices (KAP) questionnaire among student Tuberculosis (TB) Patients (STBP-KAPQ) in China. BMC infectious diseases, 18(1), 213.

[8] Szymona-Pałkowska, K., Janowski, K., Pedrycz, A., Mucha, D., Ambroży, T., Siermontowski, P., Adamczuk, J., Sapalska, M., Mucha, D., \& Kraczkowski, J. (2016). Knowledge of the Disease, Perceived Social Support, and Cognitive Appraisals in Women with Urinary Incontinence. BioMed research international, 2016, 3694792.

[9] Hung L. S. (2003). The SARS epidemic in Hong Kong: what lessons have we learned?. Journal of the Royal Society of Medicine, 96(8), 374-378.

[10] Kobayashi, M. Beer, K. D., Bjork, A., Chatham-Stephens, K., Cherry, C. C., Arzoaquoi, S., Frank, W., Kumeh, O., Sieka, J., Yeiah, A., Painter, J. E., Yoder, J. S., Flannery, B., Mahoney, F., \& Nyenswah, T. G. (2015). Community Knowledge, Attitudes, and Practices Regarding Ebola Virus Disease - Five Counties, Liberia, September-October, 2014. MMWR. Morbidity and mortality weekly report, 64(26), 714-718.

[11] Geldsetzer, P. (2020). Use of rapid online surveys to assess People's perceptions during infectious disease outbreaks: a cross-sectional survey on COVID-19. Journal of medical Internet research, 22 (4), e18790.

[12] Zhong, B. L., Luo, W., Li, H. M., Zhang, Q. Q., Liu, X. G., Li, W. T., \& Li, Y. (2020). Knowledge, attitudes, and practices towards COVID-19 among Chinese residents during the rapid rise period of the COVID-19 outbreak: a quick online cross-sectional survey. International journal of biological sciences, $16(10), 1745-1752$.

[13] Chan, E. Y. Y., Huang, Z., Lo, E. S. K., Hung, K. K. C., Wong, E. L. Y., \& Wong, S. Y. S. (2020). Sociodemographic Predictors of Health Risk Perception, Attitude and Behavior Practices Associated with Health-Emergency Disaster Risk Management for Biological Hazards: The Case of COVID-19 Pandemic in Hong Kong, SAR China. International Journal of Environmental Research and Public Health, 17 (11), 3869.

[14] Van, D., McLaws, M. L., Crimmins, J., MacIntyre, C. R., \& Seale, H. (2010). University life and pandemic influenza: Attitudes and intended behaviour of staff and students towards pandemic (H1N1) 2009. BMC Public Health, 10 (1), 130.

[15] Hager, E., Odetokun, I. A., Bolarinwa, O., Zainab, A., Okechukwu, O., \& Al-Mustapha, A. I. (2020). Knowledge, attitude, and perceptions towards the 2019 Coronavirus Pandemic: A bi-national survey in Africa. PloS one, 15 (7), e0236918.

[16] Azlan, A. A., Hamzah, M. R., Sern, T. J., Ayub, S. H., \& Mohamad, E. (2020). Public knowledge, attitudes and practices towards COVID-19: A cross-sectional study in Malaysia. Plos one, 15 (5), e0233668.

[17] Dkhar, S. A., Quansar, R., Saleem, S. M., \& Khan, S. (2020). Knowledge, attitude, and practices related to COVID-19 pandemic among social media users in J\&K, India. Indian journal of public health, 64 (6), 205-210.

[18] Xu, H., Mendez, M. J. G., Guo, L., Chen, Q., Zheng, L., Chen, P. \& Qiao, Y. (2020). Knowledge, Awareness, and Attitudes Relating to the COVID-19 Pandemic Among Different Populations in Central China: Cross-Sectional Survey. Journal of medical Internet research, 22 (10), e22628.

[19] Hezima, A., Aljafari, A., Aljafari, A., Mohammad, A., \& Adel, I. (2020). Knowledge, attitudes, and practices of Sudanese residents towards COVID-19. Eastern Mediterranean Health Journal, 26 (6), 646-651.

[20] Roy, D., Tripathy, S., Kar, S. K., Sharma, N., Verma, S. K., \& Kaushal, V. (2020). Study of knowledge, attitude, anxiety \& perceived mental healthcare need in Indian population during COVID-19 pandemic. Asian Journal of Psychiatry, 51,102083. 
[21] Peng, Y., Pei, C., Zheng, Y., Wang, J., Zhang, K., Zheng, Z., \& Zhu, P. (2020). A cross-sectional survey of knowledge, attitude and practice associated with COVID-19 among undergraduate students in China. BMC public health, 20 (1), $1-8$.

[22] Honarvar, B., Lankarani, K. B., Kharmandar, A., Shaygani, F., Zahedroozgar, M., Haghighi, M. R. R.,... \& Hashemi, S. M. (2020). Knowledge, attitudes, risk perceptions, and practices of adults toward COVID-19: a population and field-based study from Iran. International journal of public health, 65 (6), 731-739.

[23] Kotian, R. P., Faujdar, D., Kotian, S. P., \& D'souza, B. (2020). Knowledge and understanding among medical imaging professionals in India during the rapid rise of the covid-19 pandemic. Health and Technology, 1-6.

[24] Kamara, S., Walder, A., Duncan, J., Kabbedijk, A., Hughes, P., \& Muana, A. (2017). Mental health care during the Ebola virus disease outbreak in Sierra Leone. Bulletin of the World Health Organization, 95 (12), 842-847.

[25] Machida, M., Nakamura, I., Saito, R., Nakaya, T., Hanibuchi, T., Takamiya, T.,... \& Watanabe, H. (2020). Adoption of personal protective measures by ordinary citizens during the COVID-19 outbreak in Japan. International Journal of Infectious Diseases, 94, 139-144.

[26] Lau, L. L., Hung, N., Go, D. J., Ferma, J., Choi, M., Dodd, W., \& Wei, X. (2020). Knowledge, attitudes and practices of COVID-19 among income-poor households in the Philippines: a cross-sectional study. Journal of global health, 10 (1), 011007.

[27] Collinson, S., Khan, K., \& Heffernan, J. M. (2015). The effects of media reports on disease spread and important public health measurements. PloS one, 10 (11), e0141423.

[28] Salman, M., Mustafa, Z., Asif, N., Zaidi, H. A., Shehzadi, N., Khan, T. M. \& Hussain, K. (2020). Knowledge, attitude and preventive practices related to COVID-19 among health professionals of Punjab province of Pakistan. The Journal of Infection in Developing Countries, 14 (07), 707-712.

[29] Sari, D. K., Amelia, R., Dharmajaya, R., Sari, L. M., \& Fitri, N. K. (2020). Positive correlation between general public knowledge and attitudes regarding COVID-19 outbreak 1 Month after first cases reported in Indonesia. Journal of community health, $1-8$. 